\title{
Galileo: Close-up Views of Jupiter's Dynamic Atmosphere
}

\author{
Ashwin R. Vasavada \\ Geological and Planetary Sciences, California Institute of Technology, \\ Pasadena, CA 91125 (ashwin@gps.caltech.edu)
}

\begin{abstract}
The Galileo spacecraft's Solid State Imager (SSI) has been returning mosaics of Jupiter since 1996. The combination of high spatial resolution, broad spectral range, and short mosaic time steps has revealed a dynamic, vertically extended cloud structure not detectable by Voyager. These data have stimulated new analyses of major features such as zonal jets, the Great Red Spot, White Ovals, and the Galileo Probe entry site. Near-infrared images have provided fundamental constraints on the vertical structure of clouds and hazes, including the first imaging of a water cloud. Results from night side imaging include an extensive search for lightning, the first matching of lightning events to day side storm clouds, and the first spectral and spatial measurements of visible-wavelength auroral phenomena (not discussed here, see Vasavada et al. 2000). The identification of several tall, energetic storm systems at specific latitudes has led to new ideas about the role of moist convection in Jupiter's atmospheric energetics.
\end{abstract}

\section{Introduction}

Images of Jupiter's atmosphere have been obtained near perijove during most of Galileo's 28 eccentric orbits to date. While lacking the sheer volume of data returned by the Voyager or Cassini flyby missions (due to an antenna failure early in the mission), as an orbiter Galileo excels in its ability to observe Jupiter at high spatial resolution $\left(\approx 30 \mathrm{~km} \mathrm{pix}^{-1}\right)$ and over a multi-year time period. In addition, Galileo's SSI has a broader spectral range than Voyager's camera, extended toward the scientifically important near-infrared wavelengths.

The observing strategy for Jupiter's atmosphere has been to identify one or more relatively small regions, a few times $10^{4} \mathrm{~km}$ in dimension, as targets for each orbit. As listed in Table 1, targets have included dynamical features, geographic regions, and phenomena observable only on the night side (lightning and auroræ). Imaging sequences were designed to repeatedly capture each target within multi-image, multispectral mosaics, as Jupiter's rotation carried the target across the sunlit hemisphere. In the first orbit, for example, a $2 \times 3$-image, four-color mosaic of the Great Red Spot was acquired at times $T=0 h$ (nadir), $T=9 h$ (terminator), $T=10 h$ (nadir), and $T=11 h$ (limb) (Jupiter's rotation period is 10 hours). The combination of hourly timesteps and terminator-to-limb viewing maximized the utility of these data for both tracking cloud evolution 


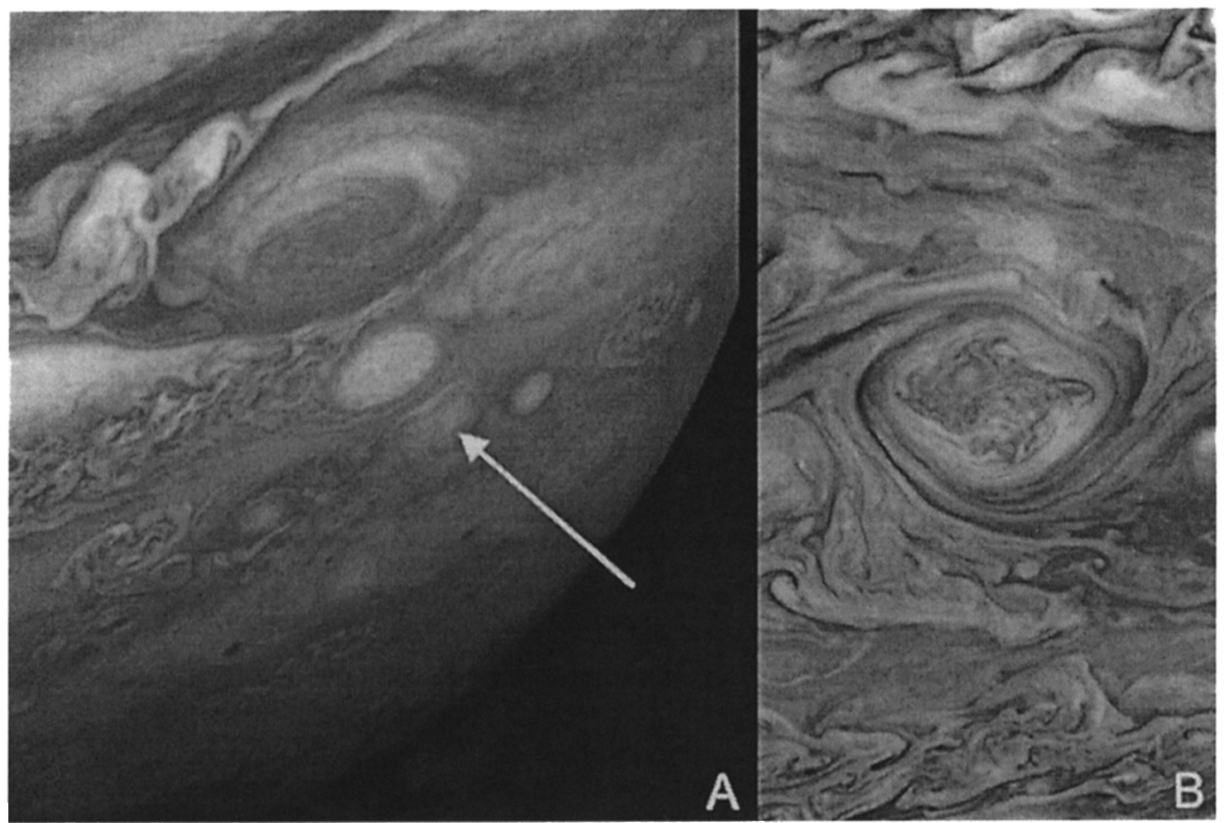

Figure 1. Jupiter's clouds as seen by Voyager I and Galileo. (a) This Voyager I view of Jupiter's southern hemisphere, showing the Great Red Spot and a White Oval directly beneath it, was obtained on Feb 25, 1979. (b) Galileo imaged this cyclonic (clockwise) vortex on May 7, 1997 as part of larger map of southern mid-latitudes. It is centered at $35^{\circ} \mathrm{S}$ latitude and has a diameter of about $5000 \mathrm{~km}$. A similar feature is marked by an arrow on the Voyager image.

and deriving vertical structure. Some situations required altogether different strategies, such as night side imaging.

\section{Dynamics of "5- $\mu \mathrm{m}$ Hotspots"}

Detailed mapping of horizontal cloud motions at several locations, including an analog to the Galileo Probe entry site, was made possible by the SSI's high spatial and temporal resolutions (Vasavada et al. 1998; Simon et al. 1998). The Probe descended into one of Jupiter's " $5-\mu \mathrm{m}$ Hotspots", dynamical features that occur $5-10^{\circ}$ north of the equator. Their low cloud opacity causes them to appear dark on optical images but bright on thermal images (such as $5-\mu \mathrm{m}$ ), which record emission escaping from decper levels. The Probe measured surprisingly low abundances of $\mathrm{NH}_{3}, \mathrm{H}_{2} \mathrm{~S}$, and $\mathrm{H}_{2} \mathrm{O}$, although values increased as the probe descended (Niemann et al. 1998; Folkner, Woo, \& Nandi 1998). The latter fact in particular suggested a meteorological explanation for the low abundances, such as dry downdrafts within hotspots, rather than a global depletion of volatiles. 
Galileo's camera helped explain this mystery by observing a similar hotspot during the fourth orbit. An analysis of cloud motions between images separated by one hour revealed a north-eastward current entering the southern boundary of the hotspot, indicative of either horizontal convergence and downwelling (Vasavada et al. 1998), or propagation of the hotspot relative to background winds (i.e., wave phenomena). Recent numerical simulations have produced coherent, nonlinear wave structures with many hotspot-like characteristics, including the observed wind patterns (Showman \& Dowling 2000). A major goal for Galileo in 2000-2001 is to re-image 5- $\mu \mathrm{m}$ hotspots.

Table 1. Targets for Atmospheric Imaging by Orbit

\begin{tabular}{llll}
\hline Orbit & Target & Orbit & Target \\
\hline G1 & Great Red Spot & E11 & N. Mid-Latitudes \\
G2 & Comet S/L-9 Impact Sites & E11 & Lightning and Auroræ \\
C3 & S. Equatorial Zone/Belt & E17 & Merged White Oval \\
C3 & N. Polar Region & C20 & N. and S. Equatorial Belts \\
C3 & Lightning and Auroræ & C20 & N.W. of Great Red Spot \\
C3 & High-Phase Limb & C20 & Jet at 24 N \\
E4 & 5- $\mu$ m Hotspot & C20 & Mesoscale Waves \\
E4 & N. Equatorial Belt & C22 & Mesoscale Waves \\
E4 & High Phase Angle & C22 & N. Polar Region \\
E6 & White Ovals & C22 & N. High Latitude \\
G7 & N. Mid-Latitudes & C22 & 5- $\mu$ m Hotspot \\
G7 & Multispectral Auroræ & C22 & Lightning \\
G8 & S. Temperate Latitudes & G28 & Great Red Spot \\
C9 & Great Red Spot & (G29) & N.W. of Great Red Spot \\
C9 & 5- $\mu$ m Hotspot & (C30) & 5- $\mu \mathrm{m}$ Hotspot \\
C9 & High Phase Angle & (I31) & Mesoscale Waves \\
C10 & N. Polar Region & (I32) & 5- $\mu$ m Hotspot \\
C10 & Lightning and Auroræ & & ()= pending \\
\hline \hline
\end{tabular}

\section{Cloud and Haze Vertical Structure}

The SSI's near-infrared imaging capability has greatly increased our understanding of the vertical distribution of aerosols. Atmospheric sounding was accomplished using three narrow-band filters that probe weak $(727 \mathrm{~nm})$ and strong $(889 \mathrm{~nm}) \mathrm{CH}_{4}$ absorption bands, and a continuum region $(756 \mathrm{~nm})$. The resulting radiances are then fit by adjusting the heights and optical depths of model cloud and haze layers. Banfield et al.'s (1998) analysis of G1-E6 data revealed the ubiquitous presence of a thin stratospheric haze (optical depth $\tau \approx 0.10$ ) and an upper tropospheric haze $(\tau \approx 2-6)$. The altitude and optical depth of the latter vary over the length scales of zonal jets and major vortices. Jupiter's distinct cloud features (and "clear" areas) are defined by horizontal variations in a vertically thin, tropospheric cloud deck with variable optical depth $(\tau \approx 0-20)$. Banfield et al. find this layer at a pressure level of $750 \pm 200 \mathrm{mb}$, close to the expected level of $\mathrm{NH}_{3}$ condensation. The properties of high-altitude hazes also have been derived from dedicated, high-phase angle images (Rages et al. 1999). 


\section{Lightning, Water Clouds, and Moist Convection}

A new view of Jupiter's global-scale dynamics and energetics has begun to emerge from several years of atmospheric image analysis. Night side images have shown that jovian lightning, while as active as terrestrial lightning, is restricted to latitude bands where the horizontal shear in the zonal flow is cyclonic (Little et al. 1999). These regions are generally clearer (which presumably indicates downwelling), but also contain chaotic cloud patterns and small, bright outbursts of cloud material. Their locations correspond with those of lightning storms observed on the night side. Multispectral analyses have shown that some lightning-bearing clouds are vertically extended, reaching from above the nominal cloud tops down to a pressure level of several bars, where water is the only reasonable condensate. Indeed, Galileo's Near-Infrared Mapping Spectrometer has measured high humidities near such features (Roos-Serote et al. 2000).

Gierasch et al. (2000) and Ingersoll et al. (2000) suggest that these are regions of vigorous moist convection on Jupiter, either vertically distinct from or contained within the Voyager-era model of (up)downwelling in the (anti)cyclonic regions. Rough calculations show that updrafts strong enough to generate these storms transport energy at a rate comparable to the planetary heat flux over their latitude band. Furthermore, these localized upwellings may form eddies at the visible cloud level that eventually transfer their momentum into the zonal jets, sustaining them.

Acknowledgments. The work reviewed in this article is the product of a very gratifying collaboration with Andy Ingersoll, Don Banfield, and Peter Gierasch. Many others, notably Blane Little, Maureen Bell, Amy Simon-Miller, and Glenn Orton contributed to the effort. The Galileo Imaging Team is led by M.J.S. Belton with camera operations run by a hard-working team at the Jet Propulsion Laboratory.

\section{References}

Banfield, D. et al. 1998, Icarus, 135, 230

Folkner, W. M. et al. 1998, JGR, 103, 22847

Gierasch, P. J. et al. 2000, Nature, 403, 628

Ingersoll, A.P. et al. 2000, Nature, 403, 630

Little, B. et al. 1999, Icarus, 142, 306

Niemann, H. B. et al. 1998, JGR, 103, 22831

Rages, K. et al. 1999, Icarus, 139, 211

Roos-Serote, M. et al. 2000, Nature, 405, 158

Showman, A.P. and T.E. Dowling 2000, Science, 289, 1737

Simon, A. A. et al. 1998, Icarus, 135, 200

Vasavada, A. R. et al. 1998, Icarus, 135, 265

Vasavada, A.R. et al. 2000, JGR, 105, 6961 